\title{
New Electrochemical (Bio)sensing Strategies Based on the Use of Dispersed Carbon Nanotubes
}

\author{
G.A. Rivas, N.F. Ferreyra, M.C. Rodríguez, M.D. Rubianes, M.L. Pedano, G.L. Luque, F.A. Gutierrez, \\ P.R. Dalmasso, A. Gasnier, E.N. Primo, Y. Jalit, F.N. Comba, M.V. Bracamonte \\ INFIQC. Departamento de Fisicoquímica. Facultad de Ciencias Químicas. Universidad Nacional de \\ Córdoba. Ciudad Universitaria. 5000 Córdoba. Argentina. \\ grivas@fcq.unc.edu.ar
}

\begin{abstract}
:
Multi-walled carbon nanotubes (MWCNTs) dispersed in different polyelectrolytes were used to develop electrochemical (bio)sensors by drop-coating of glassy carbon electrodes (GCEs). MWCNTs dispersed in polyhistidine were used for the quantification of uric acid or dopamine in the presence of large excess of ascorbic acid, and as platform to build glucose biosensors by self-assembling of glucose oxidase (GOx). The strong interaction of calf thymus-double stranded DNA (dsDNA) with the walls of bamboo-MWCNTs allowed us to obtain stable dispersions and modified GCEsfor sensitive dopamine sensing. The usefulness of GOx to disperse MWCNTs was also demonstrated. The enzyme not only disperses MWCNTs in a very efficient way, but also gives to them excellent biorecognition properties and allows to use it as platform for further immobilization of dsDNA. MWCNTs dispersed in polyethylenimine allowed the development of stable and robust electrochemical sensors for dopamine in complex mixtures and dsDNA through the surface-complex formation. In summary, the analytical performance of the (bio)sensors obtained by modification with CNT dispersions are the result of the combination of the efficiency in the dispersion of MWCNTs and the properties that the dispersing agent provide to them.
\end{abstract}

Key words: carbon nanotubes dispersion, glassy carbon electrodes, electrochemical (bio)sensors, biopolymers, bioanalytes

\section{Introduction}

Carbon nanotubes (CNTs) have been largely used for electrochemical (bio)sensing due to their outstanding mechanical, electronic and structural properties [1-3]. However, despite these unique properties, CNTs tend to aggregate in aqueous solutions due to strong $\pi$ $\pi$ interactions between their aromatic rings, making difficult their dispersion [4]. Therefore, several alternatives have been proposed to overcome this situation, the non-covalent approach being the most widely used since it does not disturb the electronic structure of the CNTs [5].

Here, we discuss the advantages of electrochemical (bio)sensors obtained by modification of glassy carbon electrodes (GCE) with multiwalled carbon nanotubes (MWCNT) dispersed in polyhistidine (Polyhis), glucose oxidase (GOx), calf-thymus double stranded DNA (dsDNA) and polyethylenimine (PEI).

\section{Experimental}

1. Preparation of the dispersions:

MWCNT-polyhistidine (MWCNT-Polyhis): The dispersion was obtained by mixing 1.00 $\mathrm{mg} / \mathrm{mLMWCNT}$ with $0.25 \mathrm{mg} / \mathrm{mL}$ Polyhis prepared with 75:25 v/v ethanol/0.200 M acetate buffer solution pH 5.00 followed by sonication for $30 \mathrm{~min}$.

MWCNT-glucose oxidase (MWCNT-GOx): The optimum dispersion was obtained by sonicating for $15 \mathrm{~min} 1.00 \mathrm{mg} / \mathrm{mL}$ CNT in $1.0 \mathrm{mg} / \mathrm{mL} \mathrm{GOx}$ solution prepared in 50:50 ethanol/water.

Bamboo-like MWCNT-calf thymus double stranded DNA: (bMWCNT-dsDNA): The dispersion was prepared by mixing $1.00 \mathrm{mg}$ of bMWCNTs with $1.00 \mathrm{~mL}$ of $100 \mathrm{ppm}$ dsDNA solution (in $50: 50 \mathrm{v} / \mathrm{v}$ ethanol/water) followed by sonication for $45 \mathrm{~min}$.

MWCNT-polyethylenimine (MWCNT-PEI): The optimum dispersion was obtained by sonicating for $15 \mathrm{~min} 1.00 \mathrm{mg} / \mathrm{mL} \mathrm{CNT}$ in $1.00 \mathrm{mg} / \mathrm{mL}$ PEI solution prepared in 50:50 v/v ethanol/water. 


\section{Preparation of glassy carbon electrodes modified with the dispersions:}

GCE/MWCNT-Polyhis, GCE/MWCNT-GOx, GCE/bMWCNT-dsDNA, and GCW/MWCNTPEI: Before modification, the GCEs were polished with alumina slurries of $1.0,0.30$, and $0.05 \mu \mathrm{m}$ for $2 \mathrm{~min}$ each. After that, they were modified by dropping $20 \mu \mathrm{L}$ of the given CNT dispersion on the top of the surfaces followed by the evaporation of the solvent by exposure to air for $90 \mathrm{~min}$.

The dispersions and the modified electrodes were evaluated by SEM, TEM, Scanning Electrochemical Microscopy (SECM), Cyclic voltammetry (CV), Amperometry, Differential Pulse Voltammetry (DPV), and Electrochemical Impedance Spectroscopy (EIS).Electrochemical experiments were performed with Epsilon (BAS) and Autolab potentiostats. Glassy carbon electrodes (GCE, CH Instruments, $3 \mathrm{~mm}$ diameter) modified with CNTs dispersed in the given polymer were used as working electrodes. A platinum wire and $\mathrm{Ag} / \mathrm{AgCl}, 3 \mathrm{M}$ $\mathrm{NaCl}$ (BAS, Model RE-5B) were used as counter and reference electrodes, respectively. All potentials are referred to the latter. The electrodes were inserted into the electrochemical cell (BAS, Model MF-1084) through holes in its Teflon cover.

The amperometric experiments were carried out in a phosphate buffer solution $(0.050 \mathrm{M}, \mathrm{pH}$ 7.40) by applying the desired potential and allowing the transient current to decay to a steady-state value prior to the addition of the analyte and the subsequent current monitoring. The cyclic voltammetric experiments were performed either in $0.200 \mathrm{M}$ acetate buffer solution $\mathrm{pH} 5.00$ or in $0.050 \mathrm{M}$ phosphate buffer solution $\mathrm{pH}$ 7.40. All the experiments were conducted at room temperature.

\section{Results and Discussion}

\section{Dispersion of MWCNTs with Polyhis}

Polyhis was successfully used for dispersingMWCNTs. Figure 1displays SEM images of glassy carbon disks modified with MWCNT-Polyhis. They reveal that the dispersion covers the whole surface although there are areas with different density of CNTs.

The modification of GCE with MWCNT-Polyhis produces a decrease of $580 \mathrm{mV}$ in the overvoltage for ascorbic acid (AA) oxidation at variance with the response observed at GCE modified just with Polyhis, where the charge transfer is more difficult due to the blocking effect of the polymer. The MWCNT-Polyhis modified GCE demonstrated to be highly stable since after 45 days storage at room temperature the response was $94.0 \%$ of the original one. GCE/MVCNT-Polyhis was successfully used in two directions: to quantify dopamine (Do) or uric acid (UA) at nanomolar levels in the presence of a large excess of AA, andas platform for building supramolecular multistructures by self-assembling of GOx on the MWCNT-Polyhis dispersion.

\section{1a. Quantification of UA and Do:}

Differential pulse voltammograms for increasing concentrations of Do from $5.0 \times 10^{-7}$ to $1.0 \times 10^{-}$

${ }^{4} \mathrm{M}$ at GCE/MWCNT-Polyhis in the presence of $1.0 \times 10^{-3} \mathrm{M}$ AA showed two well-defined peaks at $-0.060 \mathrm{~V}$ and $0.140 \mathrm{~V}$ for the oxidation of $\mathrm{AA}$ and Do, respectively. The corresponding calibration plot gave a sensitivity of (1.59 \pm $0.04) \times 10^{6} \mu \mathrm{A} / \mathrm{M},(r=0.998)$, with a linear range from $5.0 \times 10^{-7}$ to $1.0 \times 10^{-5} \mathrm{M}$, and a detection limit of $15 \mathrm{nM}$. Similarly, the DPV response for increasing concentrations of UA from $5.0 \times 10^{-7} \mathrm{M}$ to $6.0 \times 10^{-5} \mathrm{M}$ at GCE/MWCNT-Polyhis in the presence of $1.0 \mathrm{x}$ $10^{-3} \mathrm{M}$ AA presented two clearly distinguished peaks, at $-0.060 \mathrm{~V}$ for $\mathrm{AA}$ and $0.215 \mathrm{~V}$ for UA oxidation. There was a linear relationship between current and UA concentration between $5.0 \times 10^{-7}$ and $1.0 \times 10^{-5} \mathrm{M}$ UA. The average sensitivity was $(1.08 \pm 0.05) \times 10^{6} \mu \mathrm{A} / \mathrm{M},(r=$ $0.995)$ and the detection limit, $32 \mathrm{nM}$. The quantification of UA in human blood serum samples showed good agreement with the value reported by Wienner laboratory $(13.3 \%$ error), suggesting that the biosensor can be used for the determination of UA in such a complex sample as human blood serum.

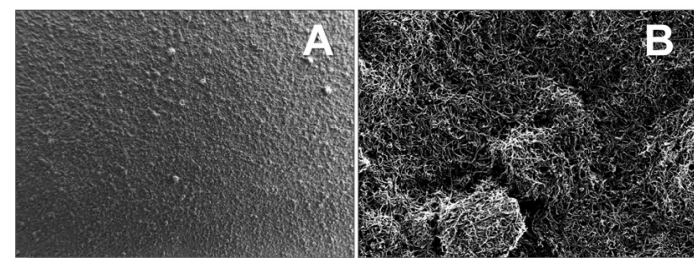

Fig. 1. SEM images of glassy carbon disks modified with dispersions of $1.00 \mathrm{mg} / \mathrm{mL}$ MWCNTs in 0.25 $\mathrm{mg} / \mathrm{mL}$ Polyhisafter $30 \mathrm{~min}$ sonication time (Magnification: A: $91 X$ and B: $8760 X$ ).

1b.Quantification of glucose:

GCE/MWCNT-Polyhis was used for the highly sensitive and selective glucose quantification. MWCNT-Polyhis dispersion demonstrated to be more efficient for the immobilization of the biocatalytic layer than the polymer alone, mainly due to the increment in the surface area provided by the presence of MWCNTs. The selected conditions for building the glucose biosensor were: adsorption of $2.0 \mathrm{mg} / \mathrm{mLGOx}$ for $5 \mathrm{~min}$, five (MWCNT-Polyhis/GOx) layers, and the presence of an outer film of $2.50 \% \mathrm{w} / \mathrm{w}$ Nafion. Fig. 2 shows the amperometric recording at $0.700 \mathrm{~V}$ for the oxidation of the 
enzymatically generated hydrogen peroxide at GCE/(MWCNT-Polyhis/GOx) $)_{5} /$ Nafion, as well as the corresponding calibration plot. The amperometric profile shows a well-defined response, with a linear relationship between oxidation current and glucose concentration in the range from $2.50 \times 10^{-4} \mathrm{M}$ to $5.00 \times 10^{-3} \mathrm{M}$ with an average sensitivity of (1.94 \pm 0.03$)$ $\mathrm{mA} / \mathrm{M}, \mathrm{r}=0.9991$ (values obtained from seven different biosensors and four different dispersions), and a detection limit of $2.2 \mu \mathrm{M}$.
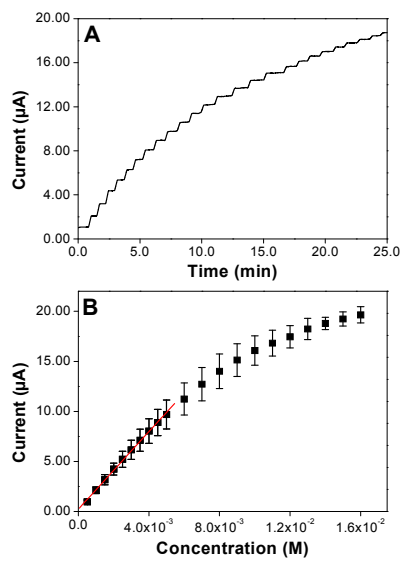

Fig. 2. (A) Amperometric response at GCE modified with five bilayers of (MWCNT-Polyhis/GOx) and one layer of Nafion for successive additions of $5.0 \times 10^{-4}$ $M$ glucose. Working potential: $0.700 \mathrm{~V}$. Calibration plot obtained from $(A)$.

The R.S.D. for 10 successive amperometric calibrations for glucose performed at $0.700 \mathrm{~V}$ using the same surface was $3.6 \%$, while the sensitivity decreased just $10 \%$ after 15 days at $4^{\circ} \mathrm{C}$. The electrode was used to quantify glucose in baby formula milk samples ("Crecer 1" milk for babies from 0 to 6 months, Argentine dairy "La Serenísima" company) previously diluted 1:40 with $0.050 \mathrm{M}$ phosphate buffer $\mathrm{pH}$ 7.40. The glucose concentration was $(3.6 \pm 0.2)$ $\times 10^{-2} \mathrm{M}$, demonstrating an excellent agreement with the value reported by "La Seresísima" company $\left(3.9 \times 10^{-2} \mathrm{M}\right)$.

\section{Dispersion of MWCNTs with GOx}

This is the first time that one enzyme is used as dispersing agent of CNTs. In this case, one interesting aspect is that GOx not only disperses the nanotubes in a very efficient way, but also gives to them, excellent biorecognition properties. The enzyme demonstrated to keep its biocatalytic activity even after dissolution in $50: 50 \mathrm{v} / \mathrm{v}$ ethanol/watersolution and sonication for 15 min using either ferrocene methanol or oxygen as redox mediators. The intimate contact of MWCNT and GOx makes possible the efficient electron transfer to the glassy carbon electrode as well as an excellent biocatalytic activity, allowing the highly sensitive detection of glucose.

The sensitivity to glucose at $0.700 \mathrm{~V}$ obtained for seventeen electrodes prepared with 6 different dispersions was $(3.2 \pm 0.2) \times 10^{2} \mu \mathrm{A} / \mathrm{M}$, $(r=0.997)$, with an R.S.D. of $6.0 \%$. The sensitivity remained highly constant after 30 days at room temperature $\left(25^{\circ} \mathrm{C}\right)$ with an average value of $(3.21 \pm 0.07) \times 10^{2} \mu \mathrm{A} / \mathrm{M}, r=$ 0.9992 .

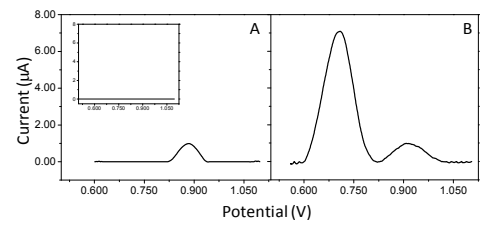

Fig. 3. Voltammetric response obtained in $0.020 \mathrm{M}$ acetate buffer solution $\mathrm{pH} 5.00$ at GCE/MWCNTGOx (A), GCE/MWCNT-GOx/PDDA (B) and bare GCE (inset)after adsorption for $20.0 \mathrm{~min}$ ( $A$ and inset) and $5.0 \mathrm{~min}(B)$ in $50.0 \mathrm{ppm}$ dsDNA solution; scan rate: $0.050 \mathrm{~V} / \mathrm{s}$.

GCE/MWCNT-GOx was also used as platform for further immobilization of biomolecules in order to develop biosensors. Calf-thymus dsDNA was immobilized at GCE/MWCNT-GOx following two strategies, direct adsorption and self-assembling with previous adsorption of the polycation polydiallyldimethylammonium (PDDA). Figure 3 displays the voltammetric response obtained in a $0.020 \mathrm{M}$ acetate buffer solution $\mathrm{pH} 5.00$ after accumulation at open circuit potential from a $50 \mathrm{ppm}$ dsDNA solution at GCE/MWCNT-GOx (A), GCE/MWCNTGox/PDDA (B) and bare GCE (inset). The presence of CNTs largely improves the adsorption and electrooxidation of dsDNA while the additional presence of the polycation layer on the top of the electrode facilitates the adsorption of dsDNA, decreasing the oxidation overvoltage and increasing drastically the associated current. The sensitivity obtained by self-assembling of dsDNA at GCE/MWCNTGOx/PDDA was $(0.15 \pm 0.02) \mu A / p p m$, which wasalmost ten times higher than the one obtained at GCE/MWCNT-GOx even after 20 min dsDNA adsorption, demonstrating the advantages of the electrostatic interaction.

\section{Dispersion of bMWCNTs with dsDNA}

Double-stranded calf thymus DNA was used for the efficient dispersion of bMWCNTs. Bamboolike CNTs present transverse walls regularly distributed along the tube which are similar to the edge-plane defects located at the end of hollow CNTs. These defects are the responsible for a facilitated charge transfer at electrodes based on bMWCNTs[6]. The TEM image showed in Fig. 4 clearly indicates that dsDNA wraps the nanostructure (Figure 4). 
Spectroscopic experiments demonstrated that the drastic treatment for dispersing the bMWCNTs (45 min sonication in a 50:50 v/vethanol/water solution), produces a partial denaturation of dsDNA that facilitates their dispersion and makes possible an efficient electron transfer to the electrode through the interaction between the DNA bases and the walls of CNTs.

Fehler! Verweisquelle konnte nicht gefunden werden.

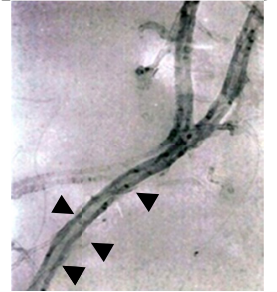

Fig. 4.TEM image obtained for bMWCNT-dsDNA. Arrows indicate the wrapping of the CNT by dsDNA.

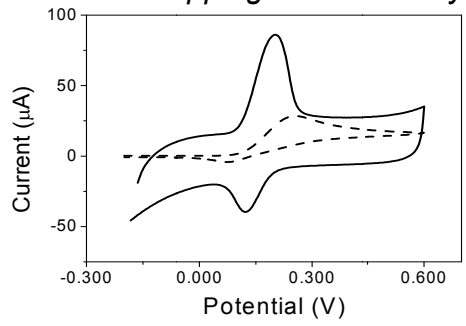

Fig. 5.Cyclic voltammograms for $1.0 \times 10^{-4} \mathrm{M}$ Do obtained at bare GCE (dashed line) and GCE/bMWCNT-dsDNA (solid line).Supporting electrolyte: $0.050 \mathrm{M}$ phosphate buffer $\mathrm{pH}$ 7.40. Scan rate: $0.100 \mathrm{~V} / \mathrm{s}$.

Figure 5 depicts cyclic voltammograms for $1.0 \mathrm{x}$ $10^{-4} \mathrm{M}$ Do at bare GCE and GCE/bMWCNTdsDNA at $0.100 \mathrm{~V} / \mathrm{s}$. It is clear that the presence of CNTs produces a drastic increase in the Do oxidation current, an improvement in the reversibility of the system as well as an important adsorption of Do at the electrode surface. Based on this important enhancement in the oxidation signal for Do, GCE/bMWCNTdsDNA was used for the quantification of Do by adsorptive stripping and DPV transduction the signal after medium exchange in $0.050 \mathrm{M}$ phosphate buffer solution $\mathrm{pH} 7.40$. After $5 \mathrm{~min}$ adsorption at open circuit potential the sensitivity for Do was almost 1000 times higher than at bare GCE $\left((3.9 \pm 0.6) \times 10^{6} \mu \mathrm{A} / \mathrm{M}\right.$ versus (3.4 \pm 0.1$\left.) \times 10^{3} \mu \mathrm{A} / \mathrm{M}\right)$, demonstrating the importance of the presence of bMWCNTdsDNA at the electrode surface as preconcentrating element. Under these conditions, the detection limit for Do obtained with the CNT-modified GCE was $50 \mathrm{nM}$.

\section{Dispersion of MWCNT with PEI}

GCE modified with MWCNTs dispersed in PEI is another interesting strategy for the development of electrochemical (bio)sensors. The highly selective and sensitive voltammetric dopamine (Do) quantification was performed in the presence of $A A$ and serotonin by using GCE/MWCNT-PEI. The ellectrocatalytic activity of MWCNT deposited on the GCE has allowed an important decrease in the overvoltages for the oxidation of $\mathrm{AA}$ and Do, making possible a clear definition of Do, serotonin and $A A$ oxidation processes. The sensitivity for Do in the presence of $1.0 \times 10^{-3} \mathrm{M} \mathrm{AA}$ and serotonin was $(2.18 \pm 0.03) \times 10^{5} \mu \mathrm{A} / \mathrm{M}(r=0.9998)$, and the detection limit for Do in the mixture was 9.2 $\times 10^{-7} \mathrm{M}$.

Linear scan voltammetry for dsDNA after adsorption at GCE/MWCNT-PEI revealed an important shifting in the oxidation peak potential due to the complex formation with PEI at the surface of the electrode. Similar behavior was obtained for short oligonucleotides. The adsorption of DNA at GCE/MWCNT-PEI is fast and it is mainly driven by electrostatic forces. The comparison of the behavior of DNA before and after thermal treatment demonstrated that the electrochemical response highly depends on the 3D structure of the nucleic acid.

\section{Conclusions}

We report different strategies for preparing electrochemical (bio)sensors based on the dispersion of multi-walled carbon nanotubes in different polymeric matrices. In all cases the ratio MWCNT/polymer and the sonication time have demonstrated to be critical aspects for the efficiency of the dispersion and the behavior of the resulting modified electrodes. The rational selection of the dispersing agent and dispersing conditions have made possible to obtain nanostructures with particular properties, that can be successfully used for the development of efficient electrochemical (bio)sensors.

\section{Acknowledgements}

The authors thank CONICET, SECYT-UNC, ANPCyT, Ministery of Science and Technology of Córdoba. FNC, ENP, MVB, YJ, FAG, and PRD acknowledge CONICET for the fellowships. AG acknowledges SECyT-UNC for the fellowships.

\section{References}

[1] S. K. Vashist, D. Zheng, K. All-Rubeaan, J. H. T. Luong, F.-S. Sheu, Biotechnology Advances 29, 169-188 (2011).

[2] C. B. Jacobs; M. J. Peairs; B. J. Venton, Analytica Chimica Acta, 662 105-127 (2010).

[3] D. Tasis, N. Tagmatarchis, A. Bianco, M. Prato, Chem. Reviews, 106 1105-1136(2006).

[4] P. D'Orazio, Clin. Chim. Acta. 412, 1749-17612011.

[5] S.W. Kim, T. Kim, Y. S. Kim, H. S. Choi, J. Lim,

[6] S. J. Yang, C. R. Park, Carbon 50 3-33 (2012).

[7] L.Y. Heng, A. Chou, J. Yu, Y.Chen, J. J. Gooding, Electrochemistry Communications 7 1457-1462 (2005) 